\title{
VOLUME GROWTH, ENTROPY AND THE GEODESIC STRETCH
}

\author{
Gerhard Knieper
}

Dedicated to Prof. W. Klingenberg on the occasion of his 70th birthday.

\section{Introduction}

Let $(M, g)$ be a compact Riemannian manifold with universal covering $\tilde{M}$. The simplest asymptotic invariant which can be associated to $(M, g)$ is the exponential growth rate $h(g)$ of volume on the universal covering, called the volume entropy. If $B_{r}^{g}(p)$ denotes the geodesic ball of radius $r$ about $p \in \tilde{M}$ and $\operatorname{vol}_{g}\left(B_{r}^{g}(p)\right)$ describes its volume with respect to the Riemannian metric $g$ lifted to $\tilde{M}$ then volume entropy is given by

$$
h(g)=\lim _{r \rightarrow \infty} \frac{\log \operatorname{vol}_{g}\left(B_{r}^{g}(p)\right)}{r} .
$$

Due to the compactness of $M$, this limit exists and is independent of $p \in \tilde{M}[17]$. Further asymptotic invariants are obtained by the dynamical properties of the geodesic flow induced by the metric $g$. For each flow invariant measure $\mu, h_{\mu}(g)$ denotes the measure theoretic entropy and $h_{\text {top }}(g)$ denotes the topological entropy of the geodesic flow. In this note we compare the volume growth and the measure theoretic entropy for a pair of metrics on the same manifold. Some of the results are similar in spirit to the entropy comparison theorems of A. Katok [12]. Crucial for the proofs will be an asymptotic comparison of the distance functions for a pair of Riemannian metrics called the geodesic stretch. Given a pair $g_{0}, g$ of Riemannian metrics on $M$, the geodesic stretch $I_{\mu}\left(g_{0}, g\right)$ measures the "stretching" of the metric $g$ relative to a reference metric $g_{0}$ and a measure $\mu$ invariant under the geodesic flow induced by $g_{0}$ (see Section 2). In the general setting, this notion has been introduced in a paper by C. Croke and A. Fathi [5], where it is called intersection, since it can be viewed as a generalization of Thurston's intersection theory of curves and measured foliation on surfaces. Using those quantities, we will prove in Section 3 the following theorem.

Received October 25, 1994 
Theorem 1.1. If $\left(M, g_{0}\right)$ is a compact space with no conjugate points and with ergodic geodesic flow, then for every metric $g$ on $M$

$$
h(g) \geq \frac{1}{I_{\mu_{L}}\left(g_{0}, g\right)} h_{\mu_{L}}\left(g_{0}\right),
$$

where $\mu_{L}$ denotes the Liouville measure induced by the metric $g_{0}$.

Remark.

a) A. Manning [17] proved that the volume entropy $h(g)$ is less or equal to the topological entropy $h_{t o p}(g)$. Equality holds in general only if the metric has no conjugate points $[9,17]$.

b) The assumption of no conjugate points is necessary for the metric $g_{0}$. For instance, on the two dimensional sphere, there exist ergodic metrics with positive measure theoretic entropy $[4,7]$ but, of course, the volume entropy is zero for every metric. However, there might be a chance to remove the assumption of ergodicity for $g_{0}$.

Let $g_{0}$ now be a metric of strictly negative curvature. If we replace the Liouville measure by the Bowen-Margulis measure $\mu_{B M}$ of $g_{0}$ (this is the unique measure of maximal entropy, i.e. $\left.h_{\mu_{B M}}\left(g_{0}\right)=h_{t o p}\left(g_{0}\right)=h\left(g_{0}\right)\right)$, we will prove Theorem 1.2 in the third Section.

Theorem 1.2. If $\left(M, g_{0}\right)$ is a compact space of negative curvature, then for every metric $g$ on $M$

$$
h(g) \geq \frac{1}{I_{\mu_{B M}}\left(g_{0}, g\right)} h\left(g_{0}\right) .
$$

If $g$ is also of negative curvature, equality holds if and only if the geodesic flows $\Phi_{g_{0}}$ of $g_{0}$ and $\Phi_{g}$ of $g$ are up to a scaling time preserving conjugate, i.e. there exists a homeomorphism $F:(S M)_{g_{0}} \rightarrow(S M)_{g}$ such that

$$
F \circ \Phi_{g_{0}}^{c t}=\Phi_{g}^{t} \circ F
$$

where $c=\frac{h(g)}{h\left(g_{0}\right)}$ and $(S M)_{g}$ denotes the unit tangent bundle with respect to $g$.

Remark.

a) The inequality in the case where both metrics are of negative curvature has been also obtained using different methods by M. Burger [3]. He shows that equality holds if and only if the marked length spectra are proportional. It is known [10] that this also implies the existence of a conjugating homeomorphism. However, so far only a sketch of his proof is available. b) Recently Besson, Courtois, and Gallot [2] proved that a locally symmetric metric of negative curvature is the unique global minimum of $h$ on the space of metrics with normalized volume. More precisely, they obtained: 
If $\left(M, g_{0}\right)$ is a compact locally symmetric space of negative curvature and $\operatorname{dim} M \geq 3$, then for all other metrics $g$ on $M$

$$
h(g) \geq h\left(g_{0}\right)\left(\frac{\operatorname{vol}_{g_{0}}(M)}{\operatorname{vol}_{g}(M)}\right)^{\frac{1}{n}},
$$

where the inequality is strict unless $g$ and $g_{0}$ are isometric up to a scaling. c) It has been proved by J.P. Otal [20] that two metrics of negative curvature on a surface with time preserving conjugacy of their geodesic flows are isometric.

Combining these results, we obtain:

Corollary 1.3. Let $M$ be a compact manifold and denote by $R_{\text {ent }}(M)$ the space of Riemannian metrics of negative curvature and with normalized entropy one. Then for all $g_{1}$ and $g_{2} \in R_{\text {ent }}(M)$

$$
I_{\mu_{B M}}\left(g_{1}, g_{2}\right) \geq 1
$$

where the inequality is strict unless there exists a time preserving conjugacy between the geodesic flows of $g_{1}$ and $g_{2}$. This conjugacy is induced by an isometry if either $\operatorname{dim} M=2$ or one of the metrics $g_{i}$ is locally symmetric and the volume of both metrics coincides.

Remark. It seems to be likely that the condition on the volumes is vacuous. For instance, if the conjugacy is $C^{1}$ the volume of the two metrics automatically coincides [6]. We conjecture that also for $\operatorname{dim} M \geq 3$ the geodesic stretch distinguishes between iso-entropy metrics of negative curvature up to isometry. By Corollary 1.3, this is equivalent to the statement that a time preserving conjugacy is induced by an isometry.

In [13], we proved that the topological entropy restricted to the metrics of negative curvature is a $C^{\infty}$ functional. In [14], we derived an explicit formula for the first derivative. Moreover, if $R_{\mathrm{vol}}(M)$ denotes the space of Riemannian metrics of negative curvature with normalized volume one, we obtained: $h: R_{\mathrm{vol}}(M) \rightarrow \mathbf{R}$ has a critical point at $g_{0} \in R_{\mathrm{vol}}(M)$ if and only if the Bowen-Margulis measure $\mu_{B M}$ and the Liouville measure $\mu_{L}$ coincide on symmetric two-forms. This leads to Corollary 1.4 proved in Section 4 .

Corollary 1.4. Let $g_{0} \in R_{\mathrm{vol}}(M)$ be a critical point of the volume entropy. Then for any other metric $g$ on $M$

$$
h(g)^{2} \geq \frac{1}{\frac{1}{n} \int_{M} t_{g_{0}} g d \operatorname{vol}_{g_{0}}} h\left(g_{0}\right)^{2},
$$


where $\operatorname{tr}_{g_{0}} g$ denotes the trace of $g$ relative to $g_{0}$ and $v l_{g_{0}}(M)$ is the volume of $M$ with respect to the metric $g_{0}$. Furthermore, equality holds if and only if $g_{0}=g$ up to a constant.

Let $g$ be a negatively curved metric on $M$ and denote by $\operatorname{Conf}(g)$ the space of Riemannian metrics conformally equivalent to $g$. Then, as we have shown in [14], $h: R_{\mathrm{vol}}(M) \cap \operatorname{Conf}(g) \rightarrow \mathbf{R}$ has a critical point $g_{0} \in$ $R_{\mathrm{vol}}(M) \cap \operatorname{Conf}(g)$ if and only if the Bowen-Margulis and the Liouville measure of $g_{0}$ coincide on functions which are constant on the fibers of $S M_{g_{0}}$.

The following statement will be proved in Section 4 .

Corollary 1.5. Let $g_{0} \in R_{\mathrm{vol}}(M) \cap \operatorname{Conf}(g)$ be a critical point of $h$. Then for all other metrics $\tilde{g} \in \operatorname{Conf}(g)$ with $\operatorname{vol}_{\tilde{g}}(M)=1$ (not necessarily of negative curvature)

$$
h(\tilde{g}) \geq h\left(g_{0}\right),
$$

and equality holds if and only if $\tilde{g}=g_{0}$. In particular, a critical point is an absolute minimum in the conformal class of metrics with nomalized volume and, therefore, the critical point is uniquely determined if it exists.

Remark.

a) If $\operatorname{Conf}(g)$ contains a locally symmetric metric then this metric is the critical point since in this case the Liouville- and the Bowen-Margulis measure coincide. In particular, for surfaces of negative curvature, as has been shown in [14], the only critical points are metrics of constant negative curvature.

b) If $\operatorname{Conf}(g)$ does not contain a locally symmetric metric, it would be interesting to compute the minimal conformal entropy given by

$$
\min h_{\operatorname{Conf}(g)}=\inf \left\{h(\tilde{g}) \mid \tilde{g} \in \operatorname{Conf}(g), \operatorname{vol}_{\tilde{g}}(M)=1\right\} .
$$

Does there exist a metric $g_{0} \in \operatorname{Conf}(g)$ with $v l_{g_{0}}(M)=1$ which admits the minimal conformal entropy?

If $\lambda^{0}(g)$ denotes the bottom of the spectrum of the Laplacian on $\tilde{M}$ with respect to $g$ and

$$
\max \lambda_{\operatorname{Conf}(g)}^{0}=\sup \left\{\lambda_{0}(\tilde{g}) \mid \tilde{g} \in \operatorname{Conf}(g), \operatorname{vol}_{\tilde{g}}(M)=1\right\},
$$

as shown in [1]

$$
\max \lambda_{\operatorname{Conf}(g)}^{0} \leq \frac{1}{4} \min h_{\operatorname{Conf}(g)}^{2} .
$$

c) Corollary 1.5 has been independently obtained by G. F. Robert [22] using convexity properties of the volume entropy. 
Before proving the theorems, we would like to begin by recalling some basic facts about the geodesic stretch (see also [5]).

\section{Geodesic stretch}

Let $M=\tilde{M} / \Gamma$ be a compact manifold, where $\tilde{M}$ denotes the universal covering and $\Gamma$ the group of covering transformations. Fix two Riemannian metrics $g_{0}$ and $g$ on $M$. For each vector $v$ in the unit tangent bundle $(S M)_{g_{0}}$ of $g_{0}$ and each $t \in \mathbf{R}$, consider the geodesic $c_{v}(s)$ with respect to the Riemannian metric $g_{0}$ and initial condition $\dot{c}_{v}(0)=v$. Denote its lift to the universal covering $\tilde{M}$ by $\tilde{c}_{v}(s)$. Let $a(v, t)=d_{g}\left(\tilde{c}_{v}(0), \tilde{c}_{v}(t)\right)$ be the distance of the endpoints of the segment $\tilde{c}_{v}(s), 0 \leq s \leq t$ with respect to the metric $g$ lifted onto $\tilde{M}$. It is an easy consequence of the triangle inequality that the map $a(v, t)$ is a subadditive cocycle for the geodesic flow $\Phi_{g_{0}}$ induced by the metric $g_{0}$, i.e.

Lemma 2.1. $a\left(v, t_{1}+t_{2}\right) \leq a\left(v, t_{1}\right)+a\left(\Phi_{g_{0}}^{t_{1}} v, t_{2}\right)$ for all $v \in S \tilde{M}$ and $t_{1}, t_{2} \in \mathbf{R}$.

The following corollary is a consequence of the subadditive ergodic theorem [24].

Corollary 2.2. Let $\mu$ be a $\Phi_{g_{0}}$ invariant probability measure. Then

$$
I_{\mu}\left(g_{0}, g, v\right):=\lim _{t \rightarrow \infty} \frac{a(v, t)}{t}
$$

exists for $\mu$ almost all $v \in(S M)_{g_{0}}$ and defines a $\mu$-integrable function on $(S M)_{g_{0}}$ invariant under the geodesic flow $\Phi_{g_{0}}$.

Moreover, we have:

$$
\int_{(S M)_{g_{0}}} I_{\mu}\left(g_{0}, g, v\right) d \mu=\lim _{t \rightarrow \infty} \frac{1}{t} \int_{(S M)_{g_{0}}} a(v, t) d \mu=\inf _{t>0} \frac{1}{t} \int_{(S M)_{g_{0}}} a(v, t) d \mu .
$$

\section{Definition 2.3.}

$$
I_{\mu}\left(g_{0}, g\right):=\int_{(S M)_{g_{0}}} I_{\mu}\left(g_{0}, g, v\right) d \mu
$$

is called the geodesic stretch of the metric $g$ relative to $g_{0}$ and the measure $\mu$. Furthermore,

$$
E_{\mu}\left(g_{0}, g\right):=\int_{(S M)_{g_{0}}} g(v, v) d \mu
$$


is called the energy with respect to $\mu, g_{0}, g$.

Lemma 2.4. With the notations above, we obtain the following inequality:

$$
\left(I_{\mu}\left(g_{0}, g\right)\right)^{2} \leq E_{\mu}\left(g_{0}, g\right)
$$

Equality implies the existence of a constant $e>0$ such that

$$
g(v, v)=e \cdot g_{0}(v, v)
$$

for almost all vectors $v$ in the tangent bundle $T M$ of $M$. If the measure is positive on open sets, equality holds if and only if $g$ and $g_{0}$ are identical up to a constant and are metrics without conjugate points.

Proof. From Corollary 2.2, the $\Phi_{g_{0}}$ invariance of $\mu$ and the fact that

$$
a(v, t) \leq \int_{0}^{t}\left\|\Phi_{g_{0}}^{s} v\right\|_{g} d s \text { for all } t>0
$$

we obtain:

$$
\begin{aligned}
I_{\mu}\left(g_{0}, g\right) & \leq \frac{1}{t} \int_{(S M)_{g_{0}}} a(v, t) d \mu \leq \frac{1}{t} \int_{(S M)_{g_{0}}} \int_{0}^{t}\left\|\Phi_{g_{0}}^{s} v\right\|_{g} d s d \mu \\
& =\int_{(S M)_{g_{0}}}\|v\|_{g} d \mu \leq\left(\int_{(S M)_{g_{0}}} g(v, v) d \mu\right)^{\frac{1}{2}} .
\end{aligned}
$$

This yields the inequality. Since the last estimate above is the CauchySchwarz inequality, equality implies $g(v, v)=e$ for a positive constant $e$ and almost all $v \in(S M)_{g_{0}}$. If the measure $\mu$ is positive on open sets, equality implies $g=e \cdot g_{0}$. Furthermore, we have:

$$
\sqrt{e}=\frac{1}{t} \int_{0}^{t}\left\|\Phi_{g_{0}}^{s} v\right\|_{g} d s=\frac{a(v, t)}{t}=\frac{d_{g}\left(\tilde{c}_{v}(0), \tilde{c}_{v}(t)\right)}{t}=\sqrt{e} \frac{d_{g_{0}}\left(\tilde{c}_{v}(0), \tilde{c}_{v}(t)\right)}{t} .
$$

Therefore, each $g_{0}$-geodesic $\tilde{c}_{v}$ on the universal covering is minimizing. Hence, there are no conjugate points. 


\section{Entropy comparison}

Since any two Riemannian metrics $g, g_{0}$ on a compact manifold $M$ are equivalent, there exists a constant $a>1$ such that for all measurable sets $A$ in the universal covering $\tilde{M}$, the estimate

$$
\frac{1}{a} \operatorname{vol}_{g_{0}}(A) \leq \operatorname{vol}_{g}(A) \leq a \operatorname{vol}_{g_{0}}(A)
$$

holds. Therefore, we can take the volume element of any metric to measure the exponential growth rate of balls on the universal covering. More precisely, we have:

Lemma 3.1. Let $g, g_{0}$ be a pair of Riemannian metrics on a compact manifold $M$. Then

$$
h(g)=\lim _{r \rightarrow \infty} \frac{\log \operatorname{vol}_{g_{0}}\left(B_{r}^{g}(p)\right)}{r},
$$

where $B_{r}^{g}(p)$ is the geodesic ball of radius $r$ about $p \in \tilde{M}$ with respect to the metric $g$.

The measure theoretic entropy $h_{\mu_{L}}(g)$ with respect to the Liouville measure can be obtained as an integral over the sum of the Lyapunov exponents. If $g$ is a metric without conjugate points, there is a geometric description. For each vector $v \in S M$, let $J_{v}(t)$ be the Jacobi tensor (i.e. $J_{v}(t)$ is a linear endomorphism of the normal space of $\dot{c}_{v}(t)$ solving the Jacobi equation) along the geodesic $c_{v}(t)$ with initial conditions $J_{v}(0)=0, J_{v}^{\prime}(0)=I d$. If $\operatorname{det} J_{v}(t)$ denotes the determinant of this endomorphism, the limit

$$
\chi(v)=\lim _{t \rightarrow \infty} \frac{1}{t} \log \operatorname{det} J_{v}(t)
$$

exists for almost all $v \in S M$ and defines an integrable function such that $h_{\mu_{L}}(g)=\int_{S M} \chi(v) d \mu_{L}$. See [9] for details.

Now we are able to prove Theorem 1.1 stated in the introduction.

Proof of Theorem 1.1. Since the geodesic flow of $g_{0}$ is ergodic with respect to $g_{0}$, we have:

$$
\lim _{t \rightarrow \infty} \frac{a(v, t)}{t}=I_{\mu_{L}}\left(g_{0}, g\right) \text { and } \lim _{t \rightarrow \infty} \frac{\log \operatorname{det} J_{v}(t)}{t}=h_{\mu_{L}}\left(g_{0}\right)
$$

for almost all $v \in(S M)_{g_{0}}$, where $J_{v}$ denotes the Jacobi tensor induced by the metric $g_{0}$ and with initial conditions as above. 
For $\epsilon>0$ and $T>0$ consider the sets

$$
A^{T, \epsilon}=\left\{v \in(S M)_{g_{0}}|| \frac{a(v, t)}{t}-I_{\mu_{L}}\left(g_{0}, g\right) \mid<\epsilon, t \geq T\right\},
$$

and

$$
B^{T, \epsilon}=\left\{v \in(S M)_{g_{0}}|| \frac{\log \operatorname{det} J_{v}(t)}{t}-h_{\mu_{L}}\left(g_{0}\right) \mid<\epsilon, t \geq T\right\} .
$$

For all $\delta, 0<\delta<1$ and $\epsilon>0$ we can find a $T(\epsilon, \delta)$ such that

$$
\mu_{L}\left(A^{T, \epsilon}\right) \geq 1-\delta \quad \text { and } \quad \mu_{L}\left(B^{T, \epsilon}\right) \geq 1-\delta .
$$

Since $\mu_{L}$ is a probability measure, we have that $\mu_{L}\left(A^{T, \epsilon} \cap B^{T, \epsilon}\right) \geq 1-2 \delta$.

Using the canonical decomposition of the Liouville measure, we obtain for a fixed $\delta<\frac{1}{2}$ :

$$
\forall \epsilon>0, \exists p \in M \text { such that } \theta_{p}\left(A_{p}^{T, \epsilon} \cap B_{p}^{T, \epsilon}\right)>d_{1}>0
$$

for a constant $d_{1}$, where $\theta_{p}$ is the canonical measure of the fiber $S_{p} M$ and $A_{p}^{T, \epsilon}=A^{T, \epsilon} \cap S_{p} M, B_{p}^{T, \epsilon}=B^{T, \epsilon} \cap S_{p} M$. Given $v \in A_{p}^{T, \epsilon}$

$$
d_{g}\left(\tilde{c}_{v}(0), \tilde{c}_{v}(t)\right)=a(v, t) \leq\left(\epsilon+I_{\mu_{L}}\left(g_{0}, g\right)\right) t
$$

and, therefore, we obtain for $\tilde{p}=\tilde{c}_{v}(0) \in \tilde{M}$

$$
\begin{aligned}
B_{g_{0}}^{T, \epsilon}(\tilde{p}, t) & :=\left\{\exp _{\tilde{p}}(v s) \mid v \in A_{p}^{T, \epsilon} \cap B_{p}^{T, \epsilon}, T \leq s \leq t\right\} \\
& \subseteq B_{g}\left(\tilde{p},\left(\epsilon+I_{\mu_{L}}\left(g_{0}, g\right)\right) t\right)
\end{aligned}
$$

for all $t \geq T$.

$v \in B_{p}^{T, \epsilon}$ implies:

$$
\log \operatorname{det} J_{v}(t) \geq\left(h_{\mu_{L}}-\epsilon\right) t \quad \text { for all } \quad t \geq T .
$$

Integrate this relation; there exists a constant $d_{2}$ such that

$$
\begin{aligned}
d_{2} \cdot e^{\left(h_{\mu_{L}}-\epsilon\right) t} & \leq \int_{T}^{t} \int_{A_{p}^{T, \epsilon} \cap B_{p}^{T, \epsilon}} \operatorname{det} J_{v}(s) d \theta_{p}(v) d s \\
& =\operatorname{vol}_{g_{0}}\left(B_{g_{0}}^{T, \epsilon}(\tilde{p}, t)\right. \\
& \leq \operatorname{vol}_{g_{0}} B_{g}\left(\tilde{p},\left(\epsilon+I_{\mu_{L}}\left(g_{0}, g\right)\right) t\right),
\end{aligned}
$$


and, therefore, using Lemma 3.1

$$
\begin{aligned}
h_{\mu_{L}}-\epsilon & \leq \lim _{t \rightarrow \infty} \frac{\operatorname{vol}_{g_{0}} B_{g}\left(\tilde{p},\left(\epsilon+I_{\mu_{L}}\left(g_{0}, g\right)\right) t\right)}{\left(\epsilon+I_{\mu_{L}}\left(g_{0}, g\right)\right) t} \cdot \frac{\left(\epsilon+I_{\mu_{L}}\left(g_{0}, g\right)\right) t}{t} \\
& =h(g)\left(I_{\mu_{L}}\left(g_{0}, g\right)+\epsilon\right) .
\end{aligned}
$$

Since $\epsilon>0$ is arbitrary, this proves the theorem.

In order to prove the next theorems, we need to recall some basic facts about the asymptotic geometry of a simply connected space $\tilde{M}$ of negative curvature.

Given a metric $g$ of negative curvature, the ideal boundary $\tilde{M}(\infty)$ is defined to be the set of equivalence classes of asymptotic geodesic rays [8]. If one chooses a point $p \in \tilde{M}$, the map $G_{p}(g): S_{p} \tilde{M} \rightarrow \tilde{M}(\infty)$ which associates to each $v \in S_{p} M$ the geodesic ray $c_{v}(t)$ with initial condition $\dot{c}_{v}(0)=v$ is a bijection. The topology on $\tilde{M}(\infty)$ induced by $G_{p}(g)$ is called the sphere topology. It is well known that the sphere topology is independent of $p$. It is a consequence of a Lemma by M. Morse that the ideal boundary depends only on the quasi-isometric structure of $\tilde{M}$, i.e. it can be described in terms of quasi-rays.

Definition 3.2. Let $g$ be a Riemannian metric on $\tilde{M}$ with corresponding distance function $d_{g}$. A quasi-ray is a quasi-isometric map $\varphi: \mathbf{R}^{+} \rightarrow \tilde{M}$, i.e. there exists a constant $b>1$ such that

$$
\frac{1}{b}|t-s| \leq d_{g}(\varphi(t), \varphi(s)) \leq b|t-s| .
$$

A quasi-isometric map $\varphi: \mathbf{R} \rightarrow \tilde{M}$ is called a quasi-geodesic.

Theorem 3.3. (Morse Lemma) Let $g$ be a metric on $\tilde{M}$ such that the sectional curvature is bounded from above by a negative constant. For each quasi-geodesic (quasi-ray) $\varphi$, there exists a geodesic (ray) c such that the Hausdorff distance of their images is bounded by a constant $r>0$ depending only on $b$ and the upper bound of the sectional curvature. In the case of quasi-geodesics, the geodesic $c$ is unique up to parametrization. If we consider quasi-rays $\varphi$, the ray $c$ is uniquely determined if we require $\varphi(0)=c(0)$.

Proof. See [19] and [15].

Call two quasi-rays equivalent if their Hausdorff distance is bounded. From the Morse Lemma, it follows that we can identify the set of equivalence classes of asymptotic geodesic rays and the equivalence classes of 
quasi-rays. In particular, this implies that the ideal boundary of two equivalent Riemannian metrics $g_{1}$ and $g_{2}$ of negative curvature on the same manifold coincide. The sphere topology does not change, since for each $p \in \tilde{M}$, the map $G_{p}\left(g_{1}\right) \circ G_{p}^{-1}\left(g_{2}\right): S_{p} \tilde{M} \rightarrow S_{p} \tilde{M}$ is a homeomorphism.

Denote by $B(\tilde{M}(\infty))$ the set of finite Borel measures on $\tilde{M}(\infty)$. If $\Gamma$ is a discrete group acting on $\tilde{M}$ with compact quotient $M=\tilde{M} / \Gamma$, we can associate to each metric $g$ of negative curvature a family of measures $\left\{\mu_{p}^{g}\right\}_{p \in \tilde{M}}$ on $\tilde{M}(\infty)$ constructed in the following way.

Consider the Poincaré series

$$
P_{s}(x, y)=\sum_{\gamma \in \Gamma} e^{-s d_{g}(x, \gamma y)} .
$$

This series converges for $s>h(g)$ and diverges for $s \leq h(g)$. For a fixed $p$, a fixed $x \in \tilde{M}$ and $s>h(g)$, define a finite measure on $\tilde{M} \cup \tilde{M}(\infty)$ by

$$
\mu_{p, x}^{g}(s)=\frac{\sum_{\gamma \in \Gamma} e^{-s d_{g}(p, \gamma x)} \delta_{\gamma x}}{P_{s}(x, x)} .
$$

$\mu_{p, x}^{g}(s)$ measures the distribution of the orbit of $x$ under $\Gamma$ as viewed from $p \in \tilde{M}$. We obtain a measure at infinity if we consider a weak limit

$$
\mu_{p}^{g}=\lim _{s_{n} \rightarrow h} \mu_{p}^{g}\left(s_{n}\right),
$$

where $s_{n}>h(g)$. Since $P_{s}(x, y)$ is diverging for $s=h(g)$, it follows that $\mu_{p}^{g}$ is concentrated on the accumulation points of the orbit $\Gamma x$. These measures have the following two characterizing properties:

a) $\mu_{p}^{g}$ and $\mu_{q}^{g}$ are in the same measure class and their Radon-Nikodym derivative is for almost all $\xi \in \tilde{M}(\infty)$ given by

$$
\frac{d \mu_{p}^{g}}{d \mu_{q}^{g}}(\xi)=e^{-h(g) b_{q}(p, \xi)}
$$

where $b_{q}(p, \xi)$ is the Busemann function with respect to the metric $g$, normalized in such a way that $b_{q}(q, \xi)=0$ and $b_{q}(p, \xi)$ tends to $-\infty$ as $p$ tends to $\xi . b_{q}(p, \xi)$ can be interpreted as the signed distance between the horospheres through $p$ and $q$ with common center in $\xi$. b) The map $\mu^{g}: \tilde{M} \rightarrow B(\tilde{M}(\infty))$ is $\Gamma$-equivariant, i.e.

$$
\mu_{p}^{g} \circ \gamma=\mu_{\gamma^{-1}(p)}^{g}
$$

for all $\gamma \in \Gamma$, if $\mu_{p}^{g} \circ \gamma(A)=\mu_{p}^{g}(\gamma A)$.

One should think of $\mu^{g}$ as measuring a given set at infinity from different observation points. It turns out that the map $\mu^{g}$ with property a) and b) is 
uniquely determined up to a constant. This follows from the fact that the action of $\Gamma$ is ergodic with respect to the measure class defined by any such family $\mu^{g}$. The ergodicity of the action of $\Gamma$ on $\tilde{M}(\infty)$ can be proved along the lines of [23]. This measure class (Patterson-Sullivan measure) has been first considered by Patterson [21] in the case of Fuchsian groups and by Sullivan [23] in the study of discrete group actions on the hyperbolic space. However, the construction can be extended to general compact manifolds of negative curvature [11]. For a different representation of $\mu^{g}$ see $[16,25]$. For us the following local property of $\mu^{g}$ will be crucial [23]. Define $\operatorname{pr}_{p}^{g}$ : $\tilde{M} \rightarrow \tilde{M}(\infty)$ to be the projection of $\tilde{M}$ onto $\tilde{M}(\infty)$ by geodesic rays with respect to $g$ emanating from $p$ (the image of a set $A \subset \tilde{M}$ can be interpreted as the shadow of a set at infinity produced by a "light" source at $p$ ). For a fixed constant $R>0$, there exists a constant $c>1$ such that

$$
\frac{1}{c} e^{-h(g) d_{g}(p, x)} \leq \mu_{p}^{g}\left(p r_{p}^{g} B(x, R)\right) \leq c e^{-h(g) d_{g}(p, x)},
$$

where $B(x, R)$ is a ball of radius $R$ about $x$ for a fixed metric on $M$ equivalent to $g$. Now we will prove the first part of Theorem 1.2.

Theorem 3.4. Let $\left(M, g_{0}\right)$ be a compact manifold of negative sectional curvature. Then for every metric $g$ on $M$

$$
h(g) \geq \frac{1}{I_{\mu_{B M}}\left(g_{0}, g\right)} h\left(g_{0}\right) .
$$

Proof. Since the geodesic flow of $g_{0}$ is ergodic with respect to the BowenMargulis measure $\mu_{B M}$, we have

$$
\lim _{t \rightarrow \infty} \frac{a(v, t)}{t}=I_{\mu_{B M}}\left(g_{0}, g\right)
$$

for $\mu_{B M}$ almost all $v \in(S M)_{g_{0}}$. We remark that the triangle inequality and the fact that the two metrics are equivalent imply the existence of the limit for all vectors on a fixed weak stable manifold if it exists for one vector.

The measure $\mu_{B M}$ can be realized as

$$
d \mu_{B M}=e^{h\left(g_{0}\right) \beta_{p}(\xi, \eta)} d \mu_{p}^{g_{0}}(\xi) d \mu_{p}^{g_{0}}(\eta) d t,
$$

where $(\xi, \eta) \in(\tilde{M}(\infty) \times \tilde{M}(\infty)) \backslash$ diagonal, $\mu_{p}^{g_{0}}$ is the normalized PattersonSullivan measure with respect to $g_{0}$ and $d t$ denotes the integration along the flow lines. Furthermore, $\beta_{p}(\xi, \eta)$ is twice the Gromov product and is given by

$$
\beta_{p}(\xi, \eta)=\lim _{t \rightarrow \infty}\left(2 t-d\left(c_{p, \xi}(t), c_{p, \eta}(t)\right)\right.
$$


where $c_{p, \xi}$ is the geodesic with $c_{p, \xi}(0)=p$ and $c_{p, \xi}(\infty)=\xi$. If $v_{p}(\xi)=$ $G_{p}^{-1}\left(g_{0}\right)(\xi)=\dot{c}_{p, \xi}(0) \in S_{p} M$ is the initial vector of the geodesic $c_{p, \xi}$, the above remark and the structure of $\mu_{B M}$ imply that

$$
\lim _{t \rightarrow \infty} \frac{a\left(v_{p}(\xi), t\right)}{t}=I_{\mu_{B M}}\left(g_{o}, g\right)
$$

for $\mu_{p}^{g_{0}}$ almost all $\xi \in \tilde{M}(\infty)$. For all $\epsilon>0$ and $T>0$, we define the set

$$
A_{p}^{T, \epsilon}=\left\{\xi \in \tilde{M}(\infty)|| \frac{a\left(v_{p}(\xi), t\right)}{t}-I_{\mu_{B M}}\left(g_{0}, g\right) \mid \leq \epsilon, t \geq T\right\} .
$$

From the above statement follows that for a fixed $d \in(0,1)$ and all $\epsilon>0$, there exists $T>0$ such that $\mu_{p}^{g_{0}}\left(A_{p}^{T, \epsilon}\right) \geq d$. For $t \geq T$ consider the subset $\left\{c_{p, \xi}(t) \mid \xi \in A_{p}^{T, \epsilon}\right\}$ of the geodesic sphere $S_{t}(p)$ of radius $t$ about $p$.

Let $\left\{B\left(x_{i}, R\right) \mid i \in I\right\}$ be a covering of this subset by balls of fixed radius $R>0$ such that $x_{i} \in S_{t}(p)$ and $B\left(x_{i}, R / 4\right)$ are pairwise disjoint. Then, by the local behavior of $\mu_{p}^{g_{0}}$, there exists a constant $c>1$ independent of $t$ such that

$$
\frac{1}{c} e^{-h\left(g_{0}\right) t} \leq \mu_{p}\left(p r_{p} B\left(x_{i}, R\right)\right) \leq c e^{-h\left(g_{0}\right) t} .
$$

Obviously, $A_{p}^{T, \epsilon}$ is contained in $\bigcup_{i \in I} p r_{p} B\left(x_{i}, R\right)$ and, therefore,

$$
* \quad d \leq \mu_{p}\left(\bigcup_{i \in I} p r_{p} B\left(x_{i}, R\right)\right) \leq \sum_{i \in I} \mu_{p}\left(p r_{p} B\left(x_{i}, R\right)\right) \leq c \cdot \operatorname{card}(I) e^{-h\left(g_{0}\right) t} .
$$

From the definition of the set $A_{p}^{T, \epsilon}$, the triangle inequality, and since the metrics $g$ and $g_{0}$ are equivalent, all the balls $B\left(x_{i}, R / 4\right)$ are contained in the geodesic ball $B^{g}\left(p, c_{1}+t\left(I_{\mu_{B M}}\left(g_{0}, g\right)+\epsilon\right)\right)$ with respect to the metric $g$ for a $t$-independent constant $c_{1}$. Now we can estimate the volume of the large $B^{g}$-ball by the volume of the disjoint union $\bigcup_{i \in I} B\left(x_{i}, R / 4\right)$ and obtain, using $*$

$$
\begin{aligned}
d_{2} \cdot e^{h\left(g_{0}\right) t} & \leq \sum_{i \in I} \operatorname{vol}_{g}\left(B\left(x_{i}, R / 4\right)\right) \\
& \leq \operatorname{vol}_{g} B^{g}\left(p, t\left(I_{\mu_{B M}}\left(g_{0}, g\right)+\epsilon\right)+c_{1}\right),
\end{aligned}
$$

where $d_{2}$ is another positive and $t$-independent constant. This concludes the proof of Theorem 3.4, by the same argument as in Theorem 1.1.

To prove the second part of Theorem 1.2, we need some preparations. 
Lemma 3.5. Let $(X, \mu)$ be a probability space, $T: X \rightarrow X$ a $\mu$-preserving ergodic transformation, and $a: X \times \mathbf{N} \rightarrow \mathbf{R}$ a measurable subadditive cocycle. Assume that there exists a constant $b>0$ such that

$$
a(x, n+l) \leq a(x, n)+a\left(T^{n} x, l\right) \leq b+a(x, n+l) .
$$

Then there is a constant $a \in \mathbf{R}$ such that for $\mu$-almost all $x \in X$ we can find a sequence $n_{j} \rightarrow \infty$ such that

$$
\left|a\left(x, n_{j}\right)-n_{j} a\right| \leq c=2 b .
$$

Proof. We have the impression that Lemma 3.5 is known [3] but since we did not find a reference for the proof, we include a proof in the appendix.

Let $\left(M, g_{0}\right)$ be a compact manifold of negative curvature and $g$ any other metric. Consider two points $p, q \in \tilde{M}$ and the geodesic segment $c_{g_{0}}$ : $I \rightarrow \tilde{M}$ with respect to $g_{0}$ connecting $p$ and $q$. Then, by the Lemma of M. Morse, there exists a constant $r>0$ not depending on the choice of $p$ and $q$ and a $g$-geodesic segment $c_{g}: J \rightarrow \tilde{M}$ such that the Hausdorff distances of their images is bounded by $r$. Therefore, the subadditive cocycle $a(v, t)=d_{g}\left(\pi(v), \pi \Phi_{g_{0}}^{t} v\right)$ has the property $a\left(v, t_{1}\right)+a\left(\Phi_{g_{0}}^{t_{1}} v, t_{2}\right) \leq$ $b+a\left(v, t_{1}+t_{2}\right)$ stated in the lemma with $b=2 r$. This yields the following corollary.

Corollary 3.6. If $p \in \tilde{M}$ and $\mu_{p}^{g_{0}}$ denotes the Patterson-Sullivan measure with respect to $p$ and the metric $g_{0}$, there exists a constant $L$ such that for $\mu_{p}^{g_{0}}$ almost all $\xi \in \tilde{M}$ there is a sequence $t_{n} \rightarrow \infty$ such that

$$
\left|d_{g}\left(p, \pi \Phi_{g_{0}}^{t_{n}} v_{p}(\xi)\right)-I_{\mu_{B M}}\left(g_{0}, g\right) t_{n}\right| \leq L .
$$

Proof. The proof is a consequence of Lemma 3.5 and arguments similar to those given in the proof of Theorem 3.4.

Proposition 3.7. Let $\left(M, g_{0}\right)$ be a compact manifold of negative curvature and $g$ another negatively curved metric. Then

$$
h(g)=\frac{1}{I_{\mu_{B M}}\left(g_{0}, g\right)} h\left(g_{0}\right)
$$

implies that the Patterson-Sullivan measures of $g_{0}$ and $g$ are equivalent.

Proof. Choose a point $p \in \tilde{M}$. We will find for $\mu_{p}^{g_{0}}$ almost all $\xi$ a sequence $y_{n}=\pi \Phi_{g_{0}}^{t_{n}} v_{p}(\xi)$ such that

$$
\left|d_{g}\left(p, y_{n}\right)-I_{\mu_{B M}}\left(g_{0}, g\right) t_{n}\right| \leq L .
$$


For a fixed constant $R>0$, we obtain from the local property of the Patterson-Sullivan measure $\mu_{p}^{g}$

$$
\frac{1}{c_{1}} e^{-h(g) d_{g}\left(p, y_{n}\right)} \leq \mu_{p}^{g}\left(p r_{p}^{g}\left(B\left(y_{n}, R\right)\right) \leq c_{1} e^{-h(g) d_{g}\left(p, y_{n}\right)}\right.
$$

for a constant $c_{1}>1$.

Since $\frac{h\left(g_{0}\right)}{h(g)}=I_{\mu_{B M}}\left(g, g_{0}\right)$, there is a further constant $c_{2}>1$ such that

$$
\frac{1}{c_{2}} e^{-h\left(g_{0}\right) d_{g_{0}}\left(p, y_{n}\right)} \leq \mu_{p}^{g}\left(p r_{p}^{g}\left(B\left(y_{n}, R\right)\right) \leq c_{2} e^{-h\left(g_{0}\right) d_{g_{0}}\left(p, y_{n}\right)} .\right.
$$

Choose the constant $R$ to be bigger than the maximal Hausdorff distance $r$ of corresponding geodesic rays with respect to $g_{0}$ and $g$. Then

$$
p r_{p}^{g_{0}}\left(B\left(y_{n}, R+r\right)\right) \supset p r_{p}^{g}\left(B\left(y_{n}, R\right)\right) \supset p r_{p}^{g_{0}}\left(B\left(y_{n}, R-r\right)\right) .
$$

Therefore,

$$
c_{3} e^{-h\left(g_{0}\right) d_{g_{0}}\left(p, y_{n}\right)} \geq \mu_{p}^{g_{0}}\left(\operatorname{pr}_{p}^{g_{0}}\left(B\left(y_{n}, R+r\right)\right)\right) \geq \mu_{p}^{g_{0}} p r_{p}^{g}\left(B\left(y_{n}, R\right)\right)
$$

and

$$
\mu_{p}^{g_{0}}\left(p r_{p}^{g}\left(B\left(y_{n}, R\right)\right)\right) \geq \mu_{p}^{g_{0}}\left(p r_{p}^{g_{0}}\left(B\left(y_{n}, R-r\right)\right)\right) \geq c_{4} e^{-h\left(g_{0}\right) d_{g_{0}}\left(p, y_{n}\right)}
$$

for suitable constants $c_{3}$ and $c_{4}$.

Hence,

$$
\frac{1}{c_{5}} \leq \frac{\mu_{p}^{g}\left(p r_{p}^{g}\left(B\left(y_{n}, R\right)\right)\right)}{\mu_{p}^{g_{0}}\left(p r_{p}^{g}\left(B\left(y_{n}, R\right)\right)\right)} \leq c_{5} .
$$

Since $\operatorname{pr}_{p}^{g}\left(B\left(y_{n}, R\right)\right) \rightarrow \xi$ for $n \rightarrow \infty$, the measures $\mu_{p}^{g}$ and $\mu_{p}^{g_{0}}$ are equivalent.

The following proposition can be derived from a slightly modified version of arguments given in [10]. For the sake of completeness and the benefit of the reader, we would like to include a sketch of the proof.

Proposition 3.8. If the Patterson-Sullivan measures of a pair of Riemannian metrics $g_{0}$ and $g$ are equivalent, there is up to scaling, a time preserving conjugacy of the geodesic flows with respect to $g_{0}$ and $g$.

Proof. Let $v$ be a vector in the unit tangent bundle of $g_{0}$ and $\eta=\Phi_{g_{0}}^{-\infty} v$, $\xi=\Phi_{g_{0}}^{+\infty} v$ be the endpoints of the corresponding $g_{0}$-geodesic. Then there exists a unique vector $w$ in the unit tangent bundle of $g$ such that the corresponding $g$-geodesic is joining $\eta$ and $\xi$, i.e. $\Phi_{g}^{-\infty} w=\eta, \Phi_{g}^{+\infty} w=\xi$ and that the measures $\mu_{\pi v}^{g_{0}}$ and $\mu_{\pi w}^{g}$ have the same density at $\xi \in \tilde{M}(\infty)$, i.e. $\frac{d \mu_{\pi v}^{g_{0}}}{d \mu_{\pi w}^{g}}(\xi)=1$. 
The map $F:(S \tilde{M})_{g_{0}} \rightarrow(S \tilde{M})_{g}$ defined by $F(v)=w$ is up to a scaling a time preserving conjugacy as the following argument shows. Let $v_{1}, v_{2}$ be vectors of the tangent bundle of $g_{0}$ such that $v_{2}=\Phi_{g_{0}}^{t_{0}} v_{1}, \xi=\Phi^{\infty} v_{1}$. We have

$$
e^{h\left(g_{0}\right) t_{0}}=\frac{d \mu_{\pi v_{2}}^{g_{0}}}{d \mu_{\pi v_{1}}^{g}}(\xi)=\frac{d \mu_{\pi F v_{2}}^{g_{0}}}{d \mu_{\pi F v_{1}}^{g}}(\xi)=e^{h(g) t}
$$

if $F v_{2}=\Phi_{g}^{t} F v_{1}$. Therefore, $t_{0}=\frac{h(g)}{h\left(g_{0}\right)} t$ and we get

$$
F\left(\Phi_{g_{0}}^{\frac{h(g)}{h\left(g_{0}\right)} t} v_{1}\right)=\Phi_{g}^{t} F v_{1}
$$

The $\Gamma$-equivariance of $F$ is an easy consequence of the $\Gamma$-equivariance of the measures.

The second part of Theorem 1.2 is now a consequence of the Propositions 3.7 and 3.8.

\section{Further applications}

The following results are a combination of applications of Theorem 1.1 and the first part of Theorem 1.2, and are consequences of the fact that the geodesic stretch can be estimated from above by the energy (Lemma 2.4 and [5]).

Theorem 4.1. Let $\left(M, g_{0}\right)$ be a compact n-dimensional manifold with no conjugate points and ergodic geodesic flow. Then for all other metrics $g$ on $M$

$$
h(g)^{2} \geq \frac{\operatorname{vol}_{g_{0}}(M)}{\frac{1}{n} \int_{M} \operatorname{tr}_{g_{0}} g d \operatorname{vol}_{g_{0}}} h_{\mu_{L}}\left(g_{0}\right)^{2} .
$$

If $g$ is conformally equivalent to $g_{0}$

$$
h(g) \geq\left(\frac{\operatorname{vol}_{g_{0}}(M)}{\operatorname{vol}_{g}(M)}\right)^{\frac{1}{n}} h_{\mu_{L}}\left(g_{0}\right) .
$$

Moreover, both inequalities are strict, unless $g$ and $g_{0}$ are identical up to a constant and $h_{\mu_{L}}\left(g_{0}\right)=h\left(g_{0}\right)$.

Proof. From Lemma 2.4, it follows that

$$
I_{\mu_{L}}\left(g_{0}, g\right)^{2} \leq E_{\mu_{L}}\left(g_{o}, g\right)=\int_{(S M)_{g_{0}}} g(v, v) d \mu_{L}=\frac{1}{\operatorname{vol}_{g_{0}} M} \int_{M} \frac{t r_{g_{0}} g}{n} d \operatorname{vol}_{g_{0}} .
$$


Together with Theorem 1.1, this implies:

$$
h(g)^{2} \geq \frac{1}{I_{\mu_{L}}\left(g_{0}, g\right)^{2}} h_{\mu_{L}}\left(g_{0}\right)^{2} \geq \frac{\operatorname{vol}_{g_{0}} M}{\frac{1}{n} \int_{M} t r_{g_{0}} g d \operatorname{vol}_{g_{0}}} h_{\mu_{L}}\left(g_{0}\right)^{2} .
$$

By Lemma 2.4, equality holds if and only if $g=e g_{0}$ for a positive constant $e$. But then we have $h(g)^{2}=\frac{h\left(g_{0}\right)^{2}}{e}$ and, therefore, it follows that

$$
\frac{h\left(g_{0}\right)^{2}}{e}=\frac{\operatorname{vol}_{g_{0}}(M)}{e \operatorname{vol}_{g_{0}}(M)} h_{\mu_{L}}\left(g_{0}\right)^{2}=\frac{h_{\mu_{L}}\left(g_{o}\right)^{2}}{e} .
$$

Let $g$ be conformally equivalent to $g_{0}$, i.e. $g=\varphi g_{0}$ for a function $\varphi: M \rightarrow$ R. Then it follows, using Jensen's inequality,

$$
\frac{1}{n} \frac{\int t r_{g_{0}} g d \operatorname{vol}_{g_{0}}}{\operatorname{vol}_{g_{0}} M}=\frac{\int \varphi d \operatorname{vol}_{g_{0}}}{\operatorname{vol}_{g_{0}} M} \leq\left(\frac{\int \varphi^{\frac{n}{2}} d \operatorname{vol}_{g_{0}}}{\operatorname{vol}_{g_{0}} M}\right)^{\frac{2}{n}}=\left(\frac{\operatorname{vol}_{g} M}{\operatorname{vol}_{g_{0}} M}\right)^{\frac{2}{n}}
$$

Remark. Theorem 4.1 generalizes a result by A. Katok in two ways [12, Theorem A]. We are only assuming that our reference metric $g_{0}$ has no conjugate points. Instead of comparing the measure entropy of $g_{0}$ with the topological entropy of $g$ we compare it with the smaller volume entropy.

Theorem 4.2. Let $\left(M, g_{0}\right)$ be a compact manifold of negative curvature. Then for any other metric $g$ on $M$

$$
h(g)^{2} \geq \frac{1}{\int_{(S M)_{g_{0}}} g(v, v) d \mu_{B M}\left(g_{0}\right)} h\left(g_{0}\right)^{2},
$$

where equality holds if and only if $g$ and $g_{0}$ are identical up to a constant.

Proof. The proof follows immediately from the first part of Theorem 1.2, Lemma 2.4, and the fact that the Bowen-Margulis measure is positive on open sets [18].

Corollary 4.3. Let $\left(M, g_{0}\right)$ be a compact manifold of negative curvature. If $g$ is a metric with Ric $_{g} \geq R i c_{g_{0}}$, we obtain:

$$
\int_{(S M)_{g_{0}}} g(v, v) d \mu_{B M}\left(g_{0}\right) \geq 1 .
$$

Furthermore, the second inequality is strict unless $g_{0}=g$.

Proof. This follows from the Theorem 4.2 and Bishop's inequality. 
Let $R_{\mathrm{vol}}(M)$ be the space of Riemannian metrics with negative sectional curvature and volume one. In [14], we proved, that $g_{0}$ is a critical point of $h: R_{\mathrm{vol}}(M) \rightarrow M$ if and only if

$$
\int_{(S M)_{g_{0}}} \xi(v, v) d \mu_{B M}\left(g_{0}\right)=\int_{(S M)_{g_{0}}} \xi(v, v) d \mu_{L}\left(g_{0}\right)
$$

for all symmetric two-forms $\xi$ on $M$.

Corollary 4.4. Let $g_{0}$ be a critical point of $h: R_{\mathrm{vol}}(M) \rightarrow \mathbf{R}$. Then for all metrics $g$ on $M$, it follows that

$$
h(g)^{2} \geq \frac{1}{\frac{1}{n} \int_{M} t r_{g_{0}} g d \operatorname{vol}_{g_{0}}} h\left(g_{0}\right)^{2},
$$

where equality holds if and only if the metrics are identical up to a constant.

Proof. The proof is an immediate consequence of Theorem 4.2.

For each metric $g \in R_{\mathrm{vol}}(M)$, let $\operatorname{Conf}(g)$ be the space of Riemannian metrics conformally equivalent to $g$. Then $h: R_{\mathrm{vol}}(M) \cap \operatorname{Conf}(g) \rightarrow \mathbf{R}$ has a critical point $g_{0} \in R_{\mathrm{vol}}(M) \cap \operatorname{Conf}(g)$ if and only if

$$
\int_{(S M)_{g_{0}}} f \circ \pi(v) d \mu_{B M}\left(g_{0}\right)=\int_{(S M)_{g_{0}}} f \circ \pi(v) d \mu_{L}\left(g_{0}\right)
$$

for all continuous functions $f: M \rightarrow \mathbf{R}$, where $\pi: S M_{g_{0}} \rightarrow M$ is the canonical projection [14].

This leads to the proof of Corollary 1.5 stated in the Introduction.

Corollary 4.5. Let $g_{0}$ be a critical point of $h: R_{\mathrm{vol}}(M) \cap \operatorname{Conf}(g) \rightarrow \mathbf{R}$. Then for all metrics $\tilde{g} \in \operatorname{Conf}(g)$ with $\operatorname{vol}_{\tilde{g}}(M)=1$, it follows that

$$
h(\tilde{g}) \geq h\left(g_{0}\right),
$$

where equality holds if and only if $\tilde{g}=g_{0}$. In particular, a critical point is an absolute minimum in the conformal class of metrics with nomalized volume and, therefore, the critical point is uniquely determined if it exists.

Proof. Let $\tilde{g}$ be a metric conformally equivalent to $g_{0}$, i.e., there exists a positive function $\varphi: M \rightarrow \mathbf{R}$ such that $\tilde{g}=\varphi g_{0}$. If $g_{0}$ is a critical point of $h: R_{\mathrm{vol}}(M) \cap \operatorname{Conf}(g) \rightarrow \mathbf{R}$, we have

$$
\begin{aligned}
\int_{(S M)_{g_{0}}} \tilde{g}(v, v) d \mu_{B M}\left(g_{0}\right) & =\int_{(S M)_{g_{0}}} \varphi \cdot \pi(v) d \mu_{L} \\
& =\int_{M} \varphi d \operatorname{vol}_{g_{0}} \leq\left(\int_{M} \varphi^{n / 2} d \operatorname{vol}_{g_{0}}\right)^{2 / n}=1
\end{aligned}
$$


and, therefore, the corollary is a consequence of Theorem 4.2.

Remark. This shows that in each conformal class there can be only one metric for which the Bowen-Margulis measure and Liouville measure projected onto $M$ coincide (see also [22]).

\section{Appendix}

Proof of Lemma 3.5. Since $a(x, n)$ is a subadditive cocycle,

$$
\lim _{n \rightarrow \infty} \frac{a(x, n)}{n}=a \text { for almost all } x \in X .
$$

Without loss of generality, we can assume $a=0$ (otherwise consider $a(x, n)-n a)$. For $j \in \mathbf{N}$, let

$$
A=A_{j}=\{x \in X \mid a(x, n) \geq c \text { for all } n \geq j\} .
$$

It follows that $\mu(A)=0$. If not, define $n_{A}(x)=\inf \left\{n \geq j \mid T^{n}(x) \in A\right\}$ and consider the map $T_{A}: A \rightarrow A$ given by $T_{A}(x)=T^{n_{A}(x)}(x)$. If

$$
N_{k}(x)=n_{A}(x)+n_{A}(T x)+\ldots+n_{A}\left(T_{A}^{k-1} x\right),
$$

one obtains that $\operatorname{card}\left\{1 \leq l \leq N_{k}(x) \mid T^{l} x \in A\right\} \leq k j$. Hence,

$$
\liminf _{k \rightarrow \infty} \frac{k j}{N_{k}(x)} \leq \lim _{k \rightarrow \infty} \frac{1}{N_{k}(x)} \sum_{l=0}^{N_{k}(x)} \chi_{A}\left(T^{l} x\right)=\mu(A) .
$$

On the other hand, we have, for all $x \in A, \quad a\left(x, N_{k}(x)\right) \geq k b$. This is easily obtained by induction since $a\left(x, n_{A}(x)\right) \geq c=2 b$ and

$$
\begin{aligned}
a\left(x, N_{k}(x)\right) & =a\left(x, N_{k-1}(x)\right)+a\left(T_{A}^{k-1} x, n_{A}\left(T_{A}^{k-1} x\right)\right. \\
& \geq-b+(k-1) b+2 b=k b .
\end{aligned}
$$

But then $\lim _{k \rightarrow \infty} \frac{a\left(x, N_{k}(x)\right)}{N_{k}(x)} \geq \liminf _{k \rightarrow \infty} \frac{k b}{N_{k}(x)}>0$, which contradicts the assumption.

In a similar way, one proves that

$$
A_{j}=\{x \in X \mid a(x, n) \leq-c \text { for all } n \geq j\}
$$

has measure 0 . Let $B$ be the set of all $x \in X$ which does not have the desired property, i.e.

$$
B=\left\{x \in X \mid \exists n_{0}(x) \in \mathbf{N} \text { such that }|a(x, n)| \geq c \forall n \geq n_{0}(x)\right\} .
$$


If we define $C_{l}=\{x \in X|| a(x, n) \mid \geq c, n \geq l\}$, we can write $B=\cup C_{l}$. Since $\mu\left(C_{l}\right)=0$, we have that $\mu(B)=0$. This implies the assertion of the lemma.

\section{References}

1. G. Besson, G. Courtois, S. Gallot, Le volume et l'entropie minimal des espaces localement symétriques, Invent. Math. 103 (1991), 417-445.

2. Entropies et rigidités des espaces localement symétriques de courbure strictement négative, Prépublication de l'Institut Fourier, Grenoble, no. 281, 1994.

3. M. Burger, Intersection, the Manhattan curve, and Patterson-Sullivan theory in rank 2, Int. Math. Research Notices 7 (1993), 217-225.

4. K. Burns and M. Gerber, Real analytic Bernoulli geodesic flows on $S^{2}$, Ergodic Theory Dyn. Syst. 9 (1989), 27-45.

5. C. Croke and A. Fathi, An inequality between energy and intersection, Bull. London Math. Soc. 22 (1990), 489-494.

6. C. Croke and B. Kleiner, Conjugacy and rigidity for manifolds with a parallel vector field, Preprint.

7. V. Donnay, Geodesic flow on the two-sphere, Part II: Ergodicity, Springer Lecture Notes 1342, Dynamical Systems, 112-153.

8. P. Eberlein and B. O'Neill, Visibility manifolds, Pacific J. Math. 46 (1973), 45109.

9. A. Freiré and R. Mañé, On the entropy of the geodesic flow in manifolds without conjugate points, Invent. Math. 69 (1982), 375-392.

10. U. Hamenstädt, Time preserving conjugacies of geodesic flows, Ergodic Theory Dyn. Syst. 12 (1992), 67-74.

11. V.A. Kaimanovich, Invariant measures of the geodesic flow and measures at infinity on negatively curved manifolds, Preprint

12. A. Katok, Entropy and closed geodesics, Ergodic Theory Dyn. Syst. 2 (1982), 339-367.

13. A. Katok, G. Knieper, M. Pollicott, and H. Weiss, Differentiability and analyticity of topological entropy for Anosov and geodesic flows, Invent. Math. 98 (1989), 581-597.

14. A. Katok, G. Knieper, and H. Weiss, Formulas for the derivative and critical points of topological entropy for Anosov and geodesic flows, Commun. Math. Phys. 138 (1991), 19-31.

15. W. Klingenberg, Geodätischer Fluß auf Mannigfaltigkeiten vom hyperbolischen Typ, Invent. Math. 14 (1971), 63-82.

16. G. Knieper, Spherical means on compact Riemannian manifolds of negative curvature, to appear in Diff. Geom. and Appl.

17. A. Manning, Topological entropy for geodesic flows, Ann. Math. 110 (1979), 567-573

18. G.A. Margulis, Certain measures associated with U-flows on compact Riemannian manifolds, Funct. Anal. Appl. 4 (1970), 55-67.

19. M. Morse, Geodesics on negatively curved surfaces, Trans. Amer. Math. Soc. 22 (1921), 84-100. 
20. J.P. Otal, Le spectre marqué des longueurs des surfaces à courbure négative, Ann. Math. 131 (1990), 151-162.

21. S. Patterson, The limit set of a Fuchsian group, Acta Math. 136 (1976), 241-273.

22. G.F. Robert, Distances, longeurs et entropies, Prépublication de l'Institut Fourier, no. 238 (1993).

23. D. Sullivan, The density at infinity of a discrete group of hyperbolic motions, Publ. Math. IHES 50 (1979), 171-202.

24. P. Walters, An introduction to ergodic theory, Graduate Text in Mathematics, Springer-Verlag, 1982.

25. C. Yue, Brownian motion on Anosov foliations and manifolds of negative curvature, to appear in J. Differ. Geom.

Institut für Mathematik, Universität Augsburg, 86135 Augsburg, Germany

E-mail address: g.knieper@uni-augsburg.de 